\title{
Anthropology and Ecohealth Research in Control of Diseases for Pastorals in Tanzania
}

\author{
Peter E. Mangesho*1, 2, Esron Karimuribo ${ }^{1,3}$, James E. Mlangwa ${ }^{3}$, Leonard E. Mboera ${ }^{4}$, \\ Jonathan Rushton ${ }^{5}$, Richard Kock ${ }^{5}$, Angwara Kiwara ${ }^{6}$ and Mark Rweyemamu ${ }^{1}$ \\ ${ }^{1}$ Department of Veterinary Medicine and Public Health, Sokoine University of Agriculture, Morogoro,, Southern African Center for \\ Disease Infectious Surveillance, Morogoro, United Republic of Tanzania; ${ }^{2}$ National Institute for Medical Research- Amani Medical \\ Research Center, Muheza, United Republic of Tanzania; ${ }^{3}$ Department of Veterinary Medicine and Public Health, Sokoine University \\ of Agriculture, Morogoro, Tanzania, Morogoro, United Republic of Tanzania; ${ }^{4}$ National Institute for Medical Research, Dar es Salaam, \\ United Republic of Tanzania; ${ }^{5}$ Royal Veterinary College, University of London, London, United Kingdom; ${ }^{6}$ Muhimbili University of \\ Health and Allied Sciences, Dar es Salaam, United Republic of Tanzania
}

\section{Objective}

To collect and assess indigenous knowledge and practices to manage diseases of food security as well as create opportunities to disseminate results for improving self-help.

\section{Introduction}

The burdens of poverty and disease continue to affect the livelihoods of pastoralists in Tanzania. Their knowledge of seasons and the ecosystems has evolved over years to manage human and animal health problems, including food insecurity. But, both local and global factors are putting pressure on their knowledge base and their capacity to manage health issues, this conflict has not been adequately explored nor have the synergies between indigenous and exotic knowledge.

\section{Methods}

A situational analysis using focus group discussions, interviews and observations was employed to collect preliminary data on perceived human and animal disease syndromes with high impact on food insecurity.

\section{Results}

Preliminary findings show that the changing institutional setting of land management has a big impact on pastoralist's capacity to address human and animal diseases. They had high knowledge on animal compared to human diseases. Pastoralists, for grounded reasons, still rely on herd maximization to abate poverty in the face of widespread diseases. Modern medical and veterinary systems exist but fail to well accommodate diseases amidst challenging terrains and prevalent poverty. Low knowledge on disease transmission mechanisms compel people to implement decisions relying on disease syndromic knowledge alone. Perceived risks to diseases and consequently health seeking options seem not to match disease control possibilities.

\section{Conclusions}

More disease focused ethnographic and socio-economic studies are still needed to further investigate the broader dynamics on how pastoralists and make decisions to identify and address health issues within their broader ecology 1).

\section{Keywords}

Health; Poverty; Pastoralism; Ecology; Tanzania

\section{Acknowledgments}

1. SACIDS

2. IDRC Canada Grant no. 107030-001 for funding this study.

\section{References}

[1] Young A.G. Current research on health among Tanzanian pastoralists, and future directions for improving pastoral health in East Africa. East Africa Journal of Research 1[1], 71-78. 2009.

*Peter E. Mangesho

E-mail: peter.mangesho@sacids.org 